\title{
Quantification of carboxyl groups in carbodiimide cross-linked collagen sponges
}

\author{
Frank Everaerts, ${ }^{1}$ Mark Torrianni, ${ }^{2}$ Marc Hendriks, ${ }_{1}^{1}$ Jan Feijen $^{3}$ \\ ${ }^{1}$ Medtronic Bakken Research Center, Maastricht, The Netherlands \\ ${ }^{2}$ Medtronic Heart Valves, Santa Ana, California \\ ${ }^{3}$ Department of Polymer Chemistry and Biomaterials, Faculty of Science and Technology, Institute for Biomedical \\ Technology (BMTI), University of Twente, P.O.Box, 7500 AE Enschede, The Netherlands
}

Received 25 January 2007; revised 6 March 2007; accepted 20 March 2007

Published online 26 June 2007 in Wiley InterScience (www.interscience.wiley.com). DOI: 10.1002/jbm.a.31398

\begin{abstract}
Glutaraldehyde (GA) fixation of bioprosthetic tissue is a well adapted technique, with commercial products on the market for almost 40 years. Amine groups present in tissue react with GA to form different types of cross-links. An estimation of the degree of cross-linking of the tissue can be obtained by measuring the concentration of residual amine groups, which is frequently carried out with the 2,4,6 trinitrobenzene sulphonic acid (TNBS) assay. Cross-linked tissue and collagen matrices are usually further characterized by determining their physical properties (such as the shrinkage temperature), biological properties (such as resistance to enzymatic degradation), and mechanical properties before in vivo evaluation takes place. In an effort to improve the properties of cross-linked tissue and collagen, alternative cross-linking methods have been developed. One of these methods is based on the use of water soluble carbodiimides (CDI). It is generally accepted that this cross-linking method leads only to the formation of amide linkages between tissue carboxyl and amine groups. Therefore, until recently the TNBS assay was also used to determine the degree of cross-linking of CDI crosslinked tissue and collagen. However, it cannot be excluded that after activation of carboxyl groups of tissue and collagen by CDI, these groups can react with other nucleophiles (like hydroxyl groups) present in the matrix. To obtain a better insight in the degree of cross-linking of CDI crosslinked matrices a reliable assay for quantification of residual carboxyl groups is required. Up to now such an assay was not available. In this study a new assay to determine
\end{abstract}

residual carboxyl groups in CDI cross-linked collagen matrices is presented. Reconstituted dermal bovine collagen matrices (RDBC) were cross-linked with a water soluble CDI and N-hydroxysuccinimide (NHS) and residual carboxyl groups were labeled using 5-bromomethyl fluorescein. Subsequently, the fluorescent label was released by mild hydrolysis and quantified with capillary zone electrophoresis. A calibration curve relating the concentration of carboxyl groups with peak intensities was obtained using Sephadex ${ }^{\text {TM }}$ standards with known concentrations of carboxyl groups. The concentration of carboxyl groups in unprocessed RDBC as determined with this new technique was equal to the concentration of carboxyl groups measured by amino acid analysis. On the basis of the concentration of residual carboxyl groups determined for CDI/ NHS cross-linked RDBC and RDBC, in which the amine groups were blocked with propionaldehyde before CDI/ NHS cross-linking, it was concluded that activated carboxyl groups can also react with other groups (such as hydroxyl groups) present in the matrix. This implies that the crosslink density of RDBC matrices after treatment with CDI/NHS is higher than expected on the basis of amide bond formation only, as determined by the TNBS assay. (C) 2007 Wiley Periodicals, Inc. J Biomed Mater Res 83A: 1176-1183, 2007

Key words: collagen cross-linking; carbodiimide; determination of carboxyl groups; quantification of carboxyl groups; determination of degree of cross-linking

\section{INTRODUCTION}

Cross-linking of bioprosthetic tissue is a common procedure to prevent degradation of the tissue during in vivo application. Glutaraldehyde (GA) based cross-linking is the current standard procedure for

Correspondence to: F. Everaerts; e-mail: frank.everaerts2@ planet.nl

(C) 2007 Wiley Periodicals, Inc. the production of tissue heart valves, providing the prostheses with a low incidence of thrombo-embolism and satisfactory haemodynamic performance. ${ }^{1}$ In spite of the positive effects of GA fixation, failure of these cross-linked materials in vivo may occur mainly due to inflammation and calcification. ${ }^{2-4}$ Therefore, much research has been directed to the development of alternative cross-linking procedures to overcome these deficiencies. ${ }^{1}$

Cross-linking of collagen based materials with a water-soluble carbodiimide (such as $N^{\prime}$-(3-dimethyla- 
minopropyl)-N-ethylcarbodiimide, EDC) may be a promising alternative to GA cross-linking. ${ }^{5,6}$ Carbodiimides (CDI) are classified as zero-length crosslinking agents used to mediate the formation of amide linkages between carboxyl and amine groups. ${ }^{7}$ The advantage of the use of these so called zero length cross-linking agents is that during cross-linking no additional chemical entities are introduced between the conjugating molecules. ${ }^{7-9}$ This is in contradiction to the use of GA, which leads to the formation of a variety of chemical structures after reaction with primary amines of lysine and hydroxylysine residues of tissue. ${ }^{10}$ It has been demonstrated that diffusion of GA or GA based reaction products from GA cross-linked tissue matrices causes cytotoxic effects in vivo. ${ }^{3}$

The use of zero length cross-linking agents has become more and more important for developing new biomaterials, such as collagen, for long term implantation and recently several articles have been published on biological biomaterials based on matrices cross-linked with EDC and $N$-hydroxysuccinimide (NHS). ${ }^{5,6,11-20}$

Insight in the extent of cross-linking of matrices that can be obtained using these reagents is essential for the design of matrices with optimal properties. ${ }^{1}$

The degree of cross-linking of matrices in which only amine groups are involved in the cross-linking reaction, for instance with the use of GA, is usually determined by measuring the concentration of residual amine groups by a 2,4,6 trinitrobenzene sulphonic acid (TNBS) assay. ${ }^{18,21}$ Furthermore determination of the shrinkage temperature provides indirect information about the degree of cross-linking of the material. ${ }^{18}$ The degree of cross-linking of a matrix also has an effect on its resistance to enzymatic degradation. ${ }^{18}$ In addition, in vitro cell based assays provide information about the biocompatibility of the crosslinked structures. ${ }^{15}$ For the determination of the mechanical properties of cross-linked matrices a number of tensile tests have been developed. ${ }^{13}$ The combination of these assays provides a complementary picture of the physical/chemical properties of the tissue after processing. ${ }^{18}$

It is generally accepted that during EDC based cross-linking of collagen the carboxyl groups are activated and form amide linkages with tissue amine groups. However, it was demonstrated in literature that activated carboxyl groups of hyaluronic acid are capable of forming ester linkages after reaction with hydroxyl groups. ${ }^{22}$ To study if this reaction also occurs during EDC cross-linking of collagen a reliable test for quantification of residual carboxyl groups is required.

It has been found that titration techniques could not be used to assess the concentration of carboxyl groups of collagen based materials. ${ }^{23}$ Labeling of residual carboxyl groups with dyes developed for histology proved to be a method for obtaining qualitative information. ${ }^{24}$

Several techniques have been described in literature for the determination of carboxyl groups of water soluble materials. ${ }^{24-33}$ It has been demonstrated that 5-bromomethyl fluorescein (5-BMF) in particular is a very effective labeling agent for carboxyl groups. ${ }^{28}$ In this article, we have adapted existing schemes using 5-BMF to label carboxyl groups of reconstituted dermal bovine collagen matrices (RDBC) before and after cross-linking. After release of the label by mild hydrolysis, it was quantified by using capillary zone electrophoresis (CZE) with laser induced fluorescence (LIF) detection. Using a calibration curve relating carboxyl group concentrations of Sephadex ${ }^{\mathrm{TM}}$ standards with the fluorescence signal, it was possible for the first time to accurately determine the concentration of carboxyl groups in the collagen based samples before and after cross-linking.

\section{MATERIALS AND METHODS}

\section{Materials}

All chemicals used were obtained from Sigma Aldrich (the Netherlands) and were of analytical grade. Acetonitrile, 18-crown-6 and 5 bromomethyl fluorescein (5-BMF) stock solutions $(3 \mathrm{mM})$ in acetonitrile were stored over molecular sieves, 5A (Supelco, the Netherlands) and kept at $4{ }^{\circ} \mathrm{C}$ until use.

Reconstituted dermal bovine collagen (RDBC) was obtained in lyophilized form from Coletica (France). RDBC samples with a thickness of about $3 \mathrm{~mm}$ were cut into $2 \mathrm{~cm} \times 1 \mathrm{~cm}$ samples and stored under anhydrous conditions over calciumsulphate (Drierite ${ }^{\mathrm{TM}}$ ) until use.

\section{Methods}

Amino acid analysis of RDBC

RDBC samples processed according to the method described in Table I later were characterized by amino acid analysis (Ansynth Roosendaal, the Netherlands).

General procedure for 5-bromomethyl fluorescein labeling of carboxyl groups of RDBC samples

Five samples containing $\sim 100 \mu \mathrm{g}$ each of RDBC sponge material were swollen in DMSO $(500 \mu \mathrm{L})$ in individual amber colored HPLC vials. After swelling, $96 \mu \mathrm{L}$ of $18-$ crown-6 solution ( $3 \mathrm{mM}$ in acetonitrile) was added to the DMSO swollen samples. Thereafter anhydrous potassium 
TABLE I

Modifications of RDBC Samples

\begin{tabular}{lll}
\hline & \multicolumn{1}{c}{ Label } & \multicolumn{1}{c}{ Chemical Process } \\
\hline A & Unprocessed & None \\
B & Blocked-only & Blocking of amine groups with propionaldehyde \\
$\mathrm{C}$ & Cross-linked only & Formation of cross-links after activation of carboxyl groups by EDC/NHS \\
$\mathrm{D}$ & Blocked and cross-linked & Blocking of amine groups with propionaldehyde and subsequent \\
& formation of cross-links after activation of carboxyl groups by EDC/NHS. \\
\hline
\end{tabular}

carbonate $(20 \mathrm{mg}$ ) was added to each vial. The contents were agitated on a rotary shaker followed by the addition of $500 \mu \mathrm{L}$ of 5-bromomethyl fluorescein (5-BMF) stock solution ( $3 \mathrm{mM}$ in acetonitrile). The final mixture was incubated at $40^{\circ} \mathrm{C}$, while the duration of incubation was varied over a period of $25 \mathrm{~h}$ to perform a time course study for determination of the optimal reaction times for the labeling process.

At specific times after the start of the reaction, the 5-BMF labeled RDBC samples were rinsed with $2 \mathrm{~mL}$ of acetonitrile for $1 \mathrm{~h}$. At the end of the first hour the rinse was analyzed for the presence of 5-BMF using fluorescence spectroscopy (Perkin Elmer, LS50B, Fullerton, USA with an excitation at $488 \mathrm{~nm}$ and emission at $520 \mathrm{~nm}$ ). The rinse and analysis processes were repeated at consecutive hour intervals until no fluorescence was detected in the rinse solutions.

To assess the amount of fluorescent label bound to available carboxyl moieties the label must be liberated from the substrate. To accomplish this, the washed labeled RDBC samples were blotted free of solution and transferred to amber colored HPLC vials containing $\mathrm{NaOH}$ $(1 \mathrm{~mL}, 0.1 \mathrm{M})$. Hydrolysis times were varied up to $30 \mathrm{~h}$ to establish the time required for maximal release of 5-BMF. The resultant hydrolyzates were used for the determination of the concentration of free carboxyl groups in the original RDBC samples utilizing CZE, which is described later.

\section{Capillary zone electrophorsis}

Capillary zone electrophorsis (CZE) analysis was performed on a MDQ (Beckman, Palo Alto, USA) equipped with a $488 \mathrm{~nm}$ laser induced fluorescence detector (LIF), a $520 \mathrm{~nm}$ band pass filter and a fused silica capillary $(50 \mu \mathrm{m}$ I.D., $50 \mathrm{~cm}$ from injection to detection). Pressurized injections for $20 \mathrm{~s}$ at 10 psi were used and the voltage was set at $15 \mathrm{kV}$. Analysis took place in micellar electrokinetic chromatography mode using a borate buffer $(\mathrm{pH} 9.2$, $10 \mathrm{mM}$, adjusted with $\mathrm{NaOH}$ ) containing sodium dodecyl sulphate (SDS, $10 \mathrm{mM}$ ). A total analysis time of $20 \mathrm{~min}$ per sample was used. All analyses took place within $12 \mathrm{~h}$ after completion of the hydrolysis process. This was done to minimize possible deterioration of the fluorescent signal. Analytes were stored in the dark at $4^{\circ} \mathrm{C}$ in the CZE system during the experiment. All effluent was analyzed by the LIF detector. Peak areas were calculated using internal software (Beckman, Palo Alto, USA).
Calibration of the CZE method for quantifying carboxyl groups

A calibration curve was made by labeling carboxymethyl modified Sephadex ${ }^{\mathrm{TM}}$ with known concentrations of carboxyl groups (obtained via GE Healthcare Bio-Sciences $A B$, Uppsala, Sweden). Hereto the same labeling method as described for the RDBC samples was used. It was found that the Sephadex ${ }^{\mathrm{TM}}$ beads were compatible with the organic labeling system used in the 5-BMF reaction. The detection limit was determined at a signal to noise ratio of 3 . The noise was determined using the mean peak to peak noise $(n=3)$ of the electropherogram between 7 and $9 \mathrm{~min}$ after injection of water. The reproducibility of the method was demonstrated by quantifying the concentration of carboxyl groups of unprocessed RDBC samples on 5 different days. For each experiment a new calibration curve was made after labeling of fresh Sephadex ${ }^{\mathrm{TM}}$ standards.

RDBC samples used for determining concentrations of carboxyl groups

Table I summarizes RDBC samples processed for the evaluation of the labeling method.

Blocking of amine groups of $R D B C^{20}$

RDBC samples were each submerged in 2-(morpholino) ethane sulphonic acid buffer (MES, $10 \mathrm{~mL}, 0.05 \mathrm{M}$ adjusted to $\mathrm{pH} 6.4$ with $\mathrm{NaOH}$ ) for $1 \mathrm{~h}$. Then the MES buffer was removed and $10 \mathrm{~mL}$ of a reaction mixture containing the same MES hydration buffer supplemented with propionaldehyde $(0.5 M)$ and $\mathrm{NaCNBH}_{3}(0.05 M)$ was added to each individual reaction vessel. After $4 \mathrm{~h}$, the RDBC samples were rinsed repeatedly in Di-water for a minimum of $16 \mathrm{~h}$. The blocking efficacy was determined by the use of a colorimetric TNBS assay. ${ }^{20}$ The concentration of residual carboxyl groups was determined with the 5-BMF labeling procedure as previously described.

\section{Cross-linking of RDBC samples ${ }^{20}$}

RDBC samples (blocked or unprocessed) were each immersed in MES buffer ( $10 \mathrm{~mL}, 0.2 \mathrm{M}$ adjusted to $\mathrm{pH} 5.0$ with $\mathrm{NaOH}$ for $1 \mathrm{~h}$ ). Then the MES buffer was removed and replaced with a cross-linking solution containing the same MES buffer $(10 \mathrm{~mL})$ supplemented with $N$-hydroxysuccinimide 
TABLE II

Amino Acids Expressing Free Amine, Carboxyl, and Hydroxyl Groups Present in RDBC Samples

\begin{tabular}{llcc}
\hline $\begin{array}{l}\text { Functional } \\
\text { Group }\end{array}$ & \multicolumn{1}{c}{$\begin{array}{c}\text { Amino } \\
\text { Acid }\end{array}$} & $\begin{array}{c}\text { Number/1000 } \\
\text { Amino Acids }\end{array}$ & $\begin{array}{c}\text { Functional } \\
\text { Groups / 1000 } \\
\text { Amino Acids }\end{array}$ \\
\hline $\mathrm{NH}_{2}$ & Lysine & 20 & \\
$\mathrm{COOH}$ & Hydroxylysine & 11 & 31 \\
$\mathrm{OH}$ & Glutamic acid & 78 & 122 \\
& Aspartic acid & 44 & \\
& Serine & 33 & 144 \\
\hline
\end{tabular}

(NHS, $0.12 M)$ and $N^{\prime}$-(3-dimethylaminopropyl)-N-ethylcarbodiimide, EDC (0.3M). After $4 \mathrm{~h}$ the RDBC samples were removed and rinsed repeatedly in Di-water for a minimum of $16 \mathrm{~h}$. After rinsing, samples were lyophilized and stored over Drierite $^{\mathrm{TM}}$. The concentration of residual carboxyl groups was determined using the 5-BMF labeling procedure as described earlier.

\section{Statistical analysis}

A student's $t$-test was performed on data. The acceptance criteria were that no statistical significant difference was found if the calculated $p$ value was less then 0.05 .

\section{RESULTS}

\section{Amino acid analysis of RDBC}

The concentration of amino acids in RDBC relevant for blocking and cross-linking is presented in Table II.

\section{Calibration of the CZE method for quantifying carboxyl groups}

Figure 1 shows a typical electropherogram obtained during quantification of carboxyl groups of modified Sephadex ${ }^{\mathrm{TM}}$ or RDBC.

Two peaks were obtained: a small peak (a) which migrates prior to the main peak (b). The small peak appears in all samples in the same relative ratio to the main peak. The main peak is assigned to the fluorescent label that is released from carboxyl groups of the matrices after mild hydrolysis.

Sephadex ${ }^{\mathrm{TM}}$ beads represent an ideal model system for determining the concentration of carboxyl groups of insoluble substrates because they can be used for chemical reactions in an organic milieu without destroying the matrix. A linear calibration curve was obtained using Sephadex ${ }^{\mathrm{TM}}$ with a detec- tion limit of 500 pmoles/mL. Reproducible results could be obtained ( 5 independent calibration curves were made with $p<0.01$, determined with a student's $t$-test analysis).

\section{General procedure for the labeling of carboxyl groups of RDBC samples with 5-bromomethyl fluorescein}

Figure $2 \mathrm{a}$ represents the results of the time course study for the reaction of 5-BMF with RDBC. It can be seen that the reaction of 5-BMF has reached a maximum conversion at approximately $12 \mathrm{~h}$. To assure adequate label incorporation we further adopted $16 \mathrm{~h}$ as a standard time for the labeling reaction. For these studies a hydrolysis (fluorescent label liberation) time of $24 \mathrm{~h}$ was chosen.

Figure 2(b) represents the results of the time course study for liberating the ester linked fluorescent label residue from RDBC, utilizing a $16 \mathrm{~h}$ labeling reaction time. It can be seen that maximum release of the label was obtained at hydrolysis times $>15 \mathrm{~h}$. To assure optimum yields a hydrolysis time of $20 \mathrm{~h}$ was used for further experiments.

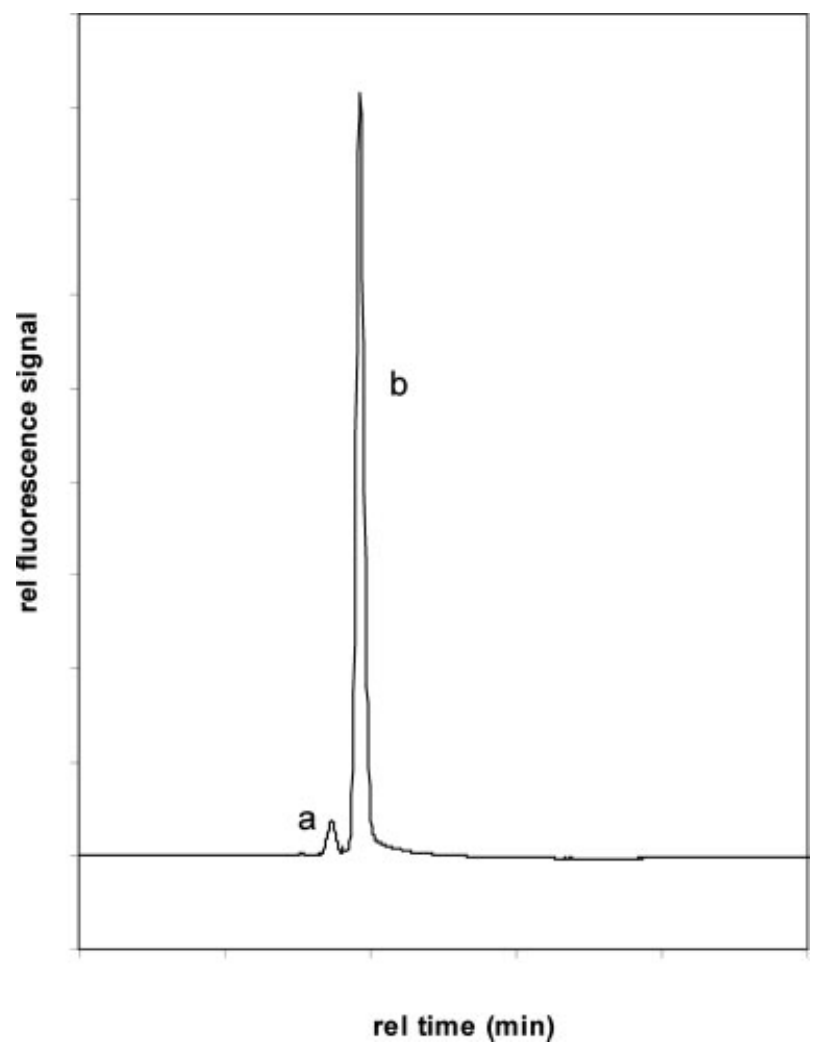

Figure 1. Electropherogram showing peaks related to the released fluorescent label after hydrolysis of labeled, Sephadex ${ }^{\mathrm{TM}}$ or unprocessed RDBC. Peak (a) is always present in the same ratio to (b) and peak (b) is used for further analysis. 

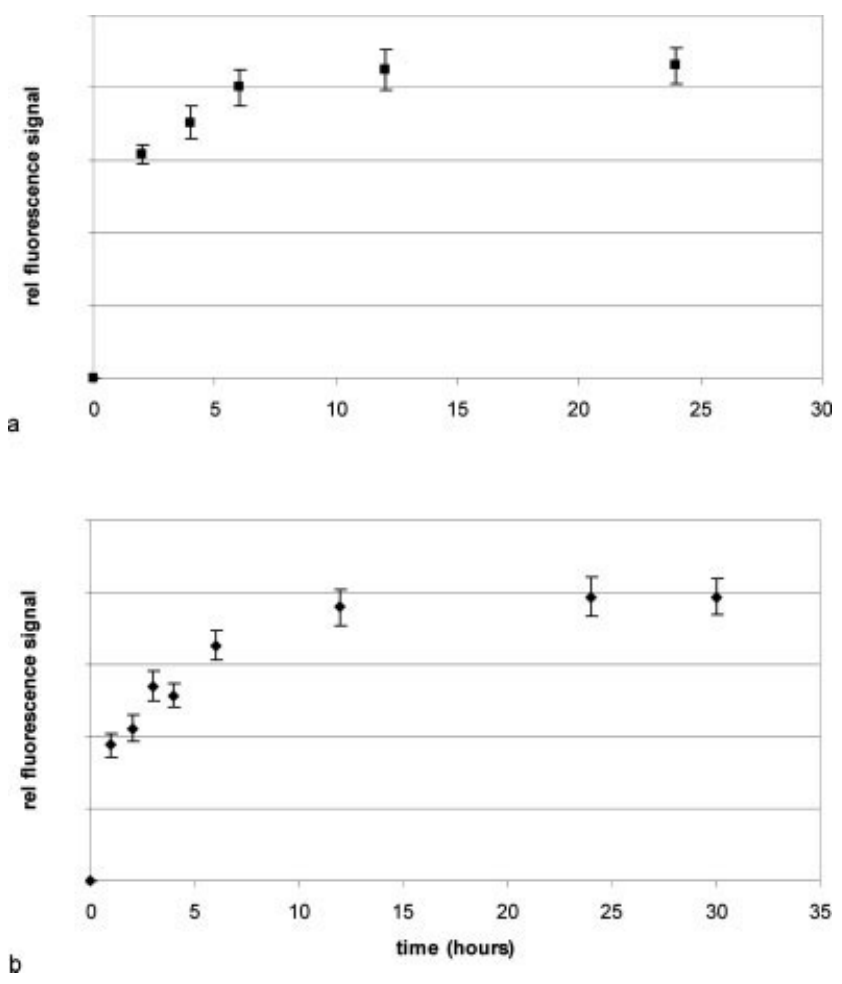

Figure 2. Single factor optimization study for the time course of fluorescent label incorporation and release from carboxyl groups in RDBC matrices. (a) shows the incorporation of label as a function of time, using a hydrolysis time of $24 \mathrm{~h}$, while (b) represents the release of the label from the matrix as a function of time, using a labeling reaction time of $16 \mathrm{~h}$.

\section{Quantification of the concentration of carboxyl groups in RDBC samples}

The amino acid analysis of the different RDBC groups (A-D in Table III) showed that the concentrations of aspartic and glutamic acid residues were the same for each group as can be expected, because for the analysis the samples are completely hydrolyzed.

No significant difference was found between the total concentration of glutamic and aspartic acid present in unprocessed RDBC as determined with amino acid analysis compared to the results obtained with the 5-BMF labeling method.

Comparing group $\mathrm{A}$ and $\mathrm{B}$ indicates that as expected blocking of amine groups does not affect the concentration of carboxyl groups. It further illustrates that the results of the 5-BMF technique are not influenced by the presence of blocked amine groups. Blocking of amine groups with propionaldehyde resulted in a significant decrease of residual amine groups as determined with the TNBS method.

After cross-linking of unprocessed RDBC $\sim 57$ carboxyl groups and 22 amine groups (per 1000 amino acid residues) are participating in cross-links. Interestingly, during cross-linking of RDBC with blocked amine groups a significant amount of carboxyl groups formed cross-links while no amine groups reacted. By comparing groups B and D it can be concluded that in both cases $\sim 36$ carboxyl groups per 1000 amino acids have formed cross-links with other reactive groups present in collagen.

\section{DISCUSSION}

Bioprosthetic devices have to be biocompatible and should not elicit cytotoxic or inflammatory responses. Although GA based cross-linking techniques are still used for most commercial products a number of different cross-linking agents for collagen based materials have been developed over the last years.

Many of these reagents react with tissue amine groups (examples are a variety of aldehydes, imidoesters, and hexamethylene diisocyanate). ${ }^{1}$

The degree of cross-linking of such materials is usually determined by a chemical analysis method using TNBS labeling of residual amine groups. ${ }^{21}$

More recently, alternative cross-linking methods for collagen based materials have been developed, in which also other groups than amine groups are

TABLE III

Comparison of Results from Amino Acid Determination and CZE Data

\begin{tabular}{lccrrrr}
\hline $\mathrm{N}=$ Number/1000 Amino Acids & $\mathrm{COOH}(\mathrm{AA})^{\mathrm{a}}$ & $\mathrm{COOH}(5-\mathrm{BMF})^{\mathrm{b}}$ & $\Delta_{\mathrm{COOH}}{ }^{\mathrm{c}}$ & $\mathrm{NH}_{2} \mathrm{TNBS}^{\mathrm{d}}$ & $\Delta_{\mathrm{NH}_{2}}{ }^{\mathrm{e}} \Delta_{\mathrm{COOH}^{-}} \Delta_{\mathrm{NH}_{2}}{ }^{\mathrm{f}}$ \\
\hline A Unprocessed & $123 \pm 3$ & $122 \pm 4$ & NA & $31 \pm 3$ & N.A. & N.A. \\
B Blocked & $121 \pm 2$ & $122 \pm 2$ & 0 & $4 \pm 2$ & $\sim 27$ & N.A. \\
C Cross-linked & $122 \pm 4$ & $65 \pm 3$ & $\sim 57$ & $9 \pm 2$ & $\sim 22$ & $\sim 35$ \\
D Blocked and Cross-linked & $121 \pm 3$ & $86 \pm 4$ & $\sim 36$ & $4 \pm 2$ & 0 & $\sim 36$ \\
\hline
\end{tabular}

N.A., not applicable.

${ }^{a}$ Carboxyl groups of aspartic and glutamic acid residues as determined by amino acid analysis.

${ }^{\mathrm{b}}$ Residual carboxyl groups as determined with 5-BMF labeling.

${ }^{c}$ Carboxyl groups involved during cross-linking.

${ }^{\mathrm{d}}$ Amine groups as determined with the TNBS colorimetric assay.

eAmine groups involved during blocking or cross-linking.

${ }^{\mathrm{f}}$ Difference in carboxyl group compared to amine group involvement in the reactions.

All values are depicted in numbers per 1000 amino acid residues. 5 samples per group per method were analyzed. 

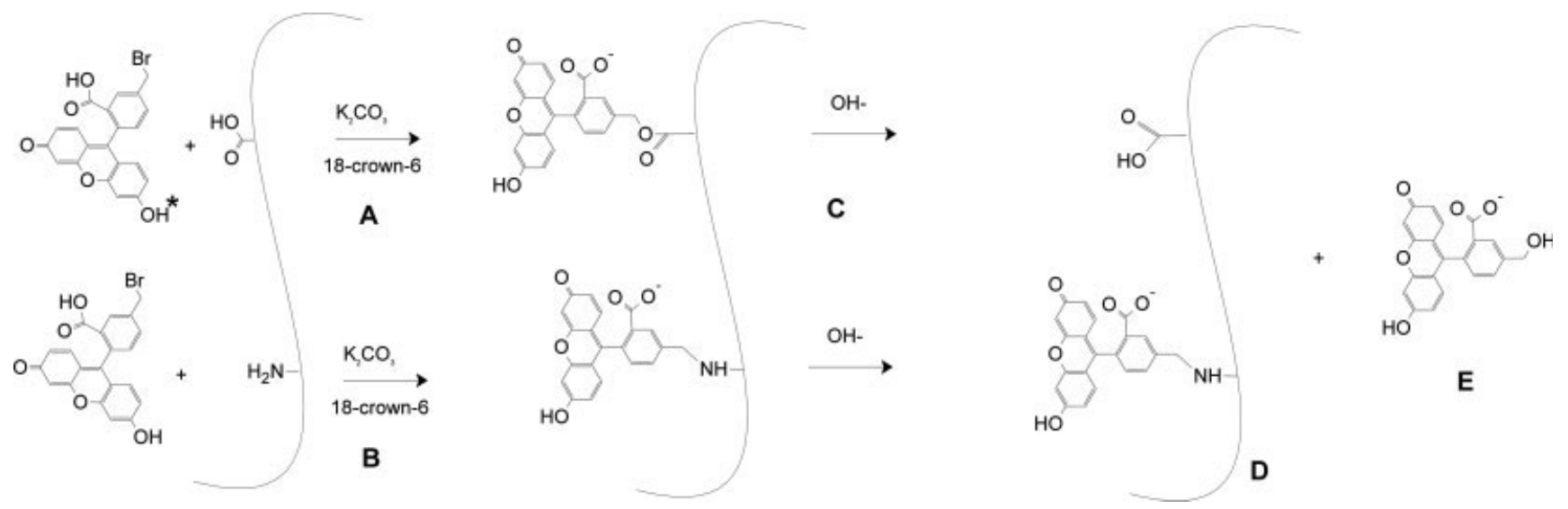

Figure 3. Reaction scheme of 5-BMF with collagen related free carboxyl and free amine groups.

involved in the cross-linking reaction. An example of such a technique is based on the involvement of carboxyl groups. Olde Damink et al. successfully demonstrated the use of $N^{\prime}$-(3-dimethylaminopropyl)- $N$-EDC (a water soluble CDI) in combination with NHS in the cross-linking of dermal sheep collagen. ${ }^{5,6,10}$ Based on promising in vivo results, published in his thesis in 1993, others have also applied water soluble EDC and NHS for the crosslinking of a wide variety of tissue matrices. ${ }^{11-20}$ One of the reasons for the interest in using EDC and NHS is that cross-linking takes place without the incorporation of new chemical entities. As a result nontoxic matrices can be obtained, which offer ideal scaffolds for cell seeding and other tissue engineering purposes. ${ }^{12,14,15}$ Other investigators have further optimized the CDI based cross-linking technology by controlling the cross-linking reactions within biological matrices. By blocking of tissue amine groups followed by incorporation of a diamine terminated spacer (Jeffamine ${ }^{\mathrm{TM}}$ ) using EDC and NHS, flexible spacers between collagen carboxyl groups could be introduced. Porcine aortic wall samples, processed in this manner showed a significantly reduced in vivo calcification as compared to porcine aortic wall samples cross-linked with EDC and NHS only. ${ }^{18,20}$

Until now it is generally accepted that during EDC based cross-linking of collagen matrices activated carboxyl groups form amide bonds with amine groups present in the matrix. ${ }^{7,9,10}$ Therefore quantification of residual amine groups with TNBS has also been used to estimate the degree of cross-linking of such matrices.

However, it cannot be excluded that activated carboxyl groups also react with other functional groups present in the matrix. This hypothesis is also based on the work of Tomihata and Ikada ${ }^{22}$ who demonstrated using FTIR that treatment of hyaluronic acid films with EDC yields cross-linked structures, rich in ester bonds.
To get a better insight in the degree of cross-linking of collagen based structures after treatment with EDC (and NHS) not only the concentration of residual amine groups has to be determined but also the concentration of remaining carboxyl groups. Up to now there is no reliable method available to quantify carboxyl groups of collagen matrices. As mentioned earlier, existing techniques based on titration of carboxyl groups or reaction of tissue samples with dyes developed for histology are cumbersome and do not have the sensitivity desired.

Because of the lack of a direct method to quantify carboxyl groups, investigators were not able to relate the actual degrees of cross-linking of collagen matrices with physical-chemical properties like the shrinkage temperature (DSC), the resistance to enzymatic degradation and the biomechanical properties. ${ }^{18,21}$

The work presented in this article is directed to the development of a reliable technique for determining the carboxyl group concentration of collagen based materials. Coletica's RDBC was chosen as a model system because its overall structure and amino acid composition are similar to other reconstituted materials on the market. RDBC was also selected because this material has a low sample to sample variation.

In this novel approach selective labeling of carboxyl groups with 5-BMF was applied. The labeling technique is a modification of the method published by Mukherjee et al. to determine the concentration of carboxyl groups of water soluble materials. ${ }^{28}$ Figure 3 depicts the reaction of 5-BMF with functional groups present in the collagen matrix.

During the reaction of 5-BMF with functional groups of collagen, a fluorescent label becomes linked to the matrix. If 5-BMF reacts with carboxyl groups, the label is attached via an ester linkage (reaction A in Fig. 3). If 5-BMF reacts with amine groups, the label is linked through an amide bond to the matrix. After labeling, residual 5-BMF is removed by wash- 
ing and part of the chemically bound fluorescent label [Fig. 3(E)] is retrieved after brief hydrolysis using $\mathrm{NaOH}(0.1 \mathrm{M})$ without damaging the remaining collagen structure. In this procedure ester linkages between the label and the matrix are cleaved, whereas the label coupled through an amine bond remains attached to the matrix [Fig. 3 (reaction $C$, product D]. This is confirmed by comparing the concentrations of carboxyl groups of unprocessed RDBC with that of amine blocked RDBC (Table III) both determined by the 5-BMF labeling procedure. The fact that the concentrations of carboxyl groups for both matrices are similar indicates that under mild hydrolytic conditions all the label bound via the ester groups will be released. By weighing the lyophilized samples before and after hydrolysis it was verified that no collagen material is lost during hydrolysis.

Mukherjee et al. ${ }^{28}$ also postulated that under the labeling conditions employed, carboxyl groups of water soluble compounds may also react with the hydroxyl group of 5-BMF (indicated with a * in Fig. 3). There is no hard evidence that this reaction takes place. If the reaction should occur also ester bonds are formed between the label and free carboxyl groups of the collagen matrix, which will also be hydrolyzed after treatment with $\mathrm{NaOH}(0.1 M)$.

Electropherograms obtained during the analyses of both processed and unprocessed RDBC show two peaks (similar to what was observed by Mukherjee et $a{ }^{28} \mathrm{~A}$ smaller leading peak was followed by a predominant peak and the ratio of the first peak to the second was always the same no matter which samples were analyzed. Spiking experiments with 5-BMF treated with $0.1 M \mathrm{NaOH}$ for times equivalent to those used to treat labeled collagen matrices resulted in similar electropherograms with the same ratio between the first and second peak. On the basis of these results the peak area of the main peak was used for determining the concentration of carboxyl groups in the original matrix.

In the course of developing this labeling process, some modifications on the method developed by Mukherjee had to be made. ${ }^{28}$ First an appropriate concentration of 5-BMF with respect to reactive groups of RDBC had to be found. To fully convert available carboxyl groups of the matrix, a molar ratio of label to all available reactive groups in excess of 2000 was utilized. This is a common practice used for many colorimetric type reactions. ${ }^{21}$

Another modification of the original 5-BMF labeling procedure concerned the reaction temperature. The optimal temperature for labeling as indicated by Mukherjee was $\sim 75^{\circ} \mathrm{C}$. It was reported that lowering the temperature to $40^{\circ} \mathrm{C}$, only decreased the labeling efficacy with a few percent. ${ }^{28}$ To avoid structural changes of the RDBC matrix, which has a shrinkage temperature of $45^{\circ} \mathrm{C}$, the labeling reaction was carried out at $40^{\circ} \mathrm{C}$.

After optimization of the reaction conditions it was found that the concentration of carboxyl groups in unprocessed RDBC as determined with this newly developed method, using Sephadex ${ }^{\mathrm{TM}}$ to prepare a calibration curve, was equal to the concentration of carboxyl groups based on aspartic and glutamic acid residues as determined with amino acid analysis (see Table III). Furthermore, as expected, prior blocking of amine groups of the RDCB matrix did not lead to changes of the concentration of carboxyl groups. Although it is generally assumed that during EDC cross-linking of collagen based materials activated carboxyl groups react only with amine groups, our results indicate that a significant part of carboxyl groups reacts with other groups of the matrix. This is also shown when RDBC samples having blocked amine groups were reacted with EDC and NHS. The data in Table III show that carboxyl groups are consumed during cross-linking. It was estimated that both for the unprocessed RDBC and RDBC having blocked amine groups $\sim 36$ carboxyl groups/1000 amino acid residues had reacted with other functional groups than amine groups. As hypothesized before, these carboxyl groups could have formed ester linkages with hydroxyl groups of the matrix. A detailed investigation on the formation of ester cross-links formed in RDBC matrices after treatment with EDC and NHS will be the subject of a forthcoming publication. Furthermore since the fiber orientation and concentration in a reconstituted collagen is different as compared to collagen present in a natural tissue, the method discussed in this article was tested on bovine pericardium, a natural collagen rich construct. Similar observations were found as discussed in this article for RDBC and results will be included in the next publication.

\section{CONCLUSION}

This study describes a new assay based on the method of Mukherjee et al. to determine the concentration of carboxyl groups of collagen containing (biological) matrices. Reconstituted collagen sponge (RDBC) was used as a model system. The concentration of carboxyl groups could be successfully determined after reacting carboxyl and amine groups of the matrix with 5-BMF followed by selective cleavage of ester bonds to release the fluorescent label from carboxyl groups. After cross-linking of RDBC with $N^{\prime}$-(3-dimethylaminopropyl)- $N$-EDC and $\mathrm{N}$-hydroxysuccinimide (NHS), it was found that based on the concentration of free carboxyl groups, that these groups had not only reacted with amine groups but also with other functional groups of the matrix. Most probably a significant percentage of the 
carboxyl groups had reacted with hydroxyl groups of the matrix to form ester cross-links.

This method provides a better determination of the cross-link density of cross-linked matrices and will help to understand the relationship between cross-link density and biophysical properties of cross-linked tissue such as shrinkage temperatures, resistance to enzymatic hydrolysis and mechanical properties. The method will also be very valuable for a better characterization and design of new matrices for bioprosthetic implants.

\section{References}

1. Hendriks, M, Everaerts F, Verhoeven M. Alternative fixation of bioprosthesis. J Long Term Eff Med Implants 2001;11:163183.

2. Speer DP, Chvapil M, Eskelson CD, Ulreich J. Biological effects of residual glutaraldehyde in glutaraldehyde-tanned collagen biomaterials. J Biomed Mater Res 1980;14:753-764.

3. Eybl E, Griesmacher A, Grimm M, Wolner E. Toxic effects of aldehydes released from fixed pericardium on bovine aortic endothelial cells. J Biomed Mater Res 1989;23:1355-1365.

4. Levy RJ. Glutaraldehyde and the calcification mechanism of bioprosthetic heart valves. J Heart Valve Dis 1994;3:101-104.

5. Olde Damink LH, Dijkstra PJ, van Luyn MJ, van Wachem PB, Nieuwenhuis P, Feijen J. Cross-linking of dermal sheep collagen using a water-soluble carbodiimide. Biomaterials 1996;17:765-773.

6. Olde Damink LH, Dijkstra PJ, van Luyn MJ, van Wachem PB, Nieuwenhuis P, Feijen J. In vitro degradation of dermal sheep collagen cross-linked using a water-soluble carbodiimide. Biomaterials 1996;17:679-684.

7. Hermanson GT. Bioconjugate Techniques, Academic Press, Inc., San Diego, CA, 1996 (ISBN 0-12-342336-8).

8. Staros JV, Wright RW, Swingle DM. Enhancement by $\mathrm{N}$-hydroxysulfosuccinimide of water soluble carbodiimidemediated coupling reactions. Anal Biochem 1986;156:220-222.

9. Sheehan JC. Carbodiimides in peptide chemistry. Am Chem Soc 1955;77:1067.

10. Olde Damink LHH. Structure and properties of cross linked dermal sheep collagen. Thesis, University of Twente, Enschede, Netherlands, 1993. ISBN 90-9005-924-5.

11. Simmons DM, Kearney JN. Evaluation of collagen crosslinking techniques for the stabilization of tissue matrices. Biotechnol Appl Biochem 1993;17(Part 1):23-29.

12. Hafemann B, Ghofrani K, Gattner HG, Stieve H, Pallua N. Cross-linking by 1-ethyl-3-(3-dimethylaminopropyl)-carbodiimide (EDC) of a collagen/elastin membrane meant to be used as a dermal substitute: Effects on physical, biochemical and biological features in vitro. J Mater Sci Mater Med 2001;12:437-446.

13. Powell HM, Boyce S. EDC cross-linking improves skin substitute strength and stability. Biomaterials 2006;27:58215827.

14. Buttafoco L, Kolkman NG, Engbers-Buijtenhuijs P, Poot AA, Dijkstra PJ, Vermes I, Feijen J. Electrospinning of collagen and elastin for tissue engineering applications. Biomaterials 2006;27:724-734; Epub 2005 Aug 19.

15. Caruso AB, Dunn MG. Changes in mechanical properties and cellularity during long-term culture of collagen fiber ACL reconstruction scaffolds. J Biomed Mater Res A 2005;73:388397.

16. Liu Y, Griffith M, Watsky MA, Forrester JV, Kuffova L, Grant D, Merrett K, Carlsson DJ. Properties of porcine and recombinant human collagen matrices for optically clear tissue engineering applications. Biomacromolecules 2006;7:1819-1828.

17. Liu Y, Gan L, Carlsson DJ, Fagerholm P, Lagali N, Watsky MA, Munger R, Hodge WG, Priest D, Griffith M. A simple, cross-linked collagen tissue substitute for corneal implantation. Invest Ophthalmol Vis Sci 2006;47:1869-1875.

18. Everaerts FJL, Torrianni M, van Luyn M, Hendriks M, Feijen J. Reduced calcification of bioprostheses, cross-linked via an improved carbodiimide based method. Biomaterials 2004;25: 5523-5530.

19. Girardot JM, Girardot MN. Amide cross-linking: an alternative to glutaraldehyde fixation. J Heart Valve Dis 1996;5:518-525.

20. Everaerts FJL, Gillissen M, Torrianni M, Zilla, P, Human P, Feijen J. Reduction of calcification of carbodiimide-processed heart valve tissue by prior blocking of amine groups with monoaldehydes. J Heart Valve Dis 2006;15:269-277.

21. Field R. Rapid determination of amino groups with TNBS. Methods Enzymol 1972;25:464-468.

22. Tomihata K, Ikada Y. Crosslinking of hyaluronic acid with watersoluble carbodiimide. J Biomed Mater Res 1997;37:243-251.

23. Kenchington A, Ward A. The titration curve of gelatin. Biochem J 1954;58:202-207.

24. http://www.invitrogen.com/search.cfm?searchTerm=Carboxylic \&category $=$ Catalog\&num $=10$ as accessed on April 14, 2006.

25. Ikeda M, Shimada K, Sakaguchi T. High-performance liquid chromatographic determination of free fatty acids with 1-naphthylamine. J Chromatogr 1983;272:251-259.

26. Durst HD, Milano M, Kikta EJ Jr, Connelly SA, Grushka EIkeda. Phenacyl esters of fatty acids via crown ether catalysts for enhanced ultraviolet detection in liquid chromatography. Anal Chem 1975;47:1797-1801.

27. Wolf HJ, Korf J. 4-bromomethyl-7-methoxycoumarin and analogues for derivatization agents for HPLC determinations. J Pharm Biomed Anal 1992;10:99-107.

28. Mukherjee PS, DeSilva KH, Karnes HT. 5-Bromomethyl fluorescein (5-BMF) for derivatization of carboxyl containing analytes for use with laser-induced fluorescence detection. Pharm Res 1995;12:930-936.

29. Zuriguel V, Caussé E, Bounéry JD, Nouadje G, Siméon N, Nertz M, Salvayre R, Couderc F. Short chain fatty acids analysis by capillary electrophoresis and indirect UV detection or laser-induced fluorescence. J Chromatogr A 1997;781:233-238.

30. Hart JJ, Welch RM, Norvell WA, Kochian LV. Measurement of thiol-containing amino acids and phytochelatin (PC2) via capillary electrophoresis with laser-induced fluorescence detection, Electrophoresis. 2002 Jan;23(1):81-7.

31. Holletz T, Moller U, Knaf A, Reinhardt R, Cech D. Synthesis and application of a new fluorescein derivative for fluorescent labeling of oligonucleotides and as a novel tool for non-radioactive DNA sequencing. Liebigs Annalen Der Chemie 1993;10:10511056.

32. Mukherjee P, Karnes H. Analysis of $\gamma$-(cholesteryloxy)butric acid in biological samples by derivatization with 5-(bromomethyl)fluorescein followed by high performance liquid chromotagraphy with laser-induced fluorescence detection. Anal Chem 1996;68:327-332.

33. Zhang Z, Liu X. Determination of jasmonic acid in bark extracts from hevea brasiliensis by capillary electrophoresis with laser-induced fluorescence detection. Anal Bioanal Chem 2005;382:1616-1619. 